\title{
RESTRICTED MEASURABILITY
}

TREVOR J. MCMINN

1. Introduction. It is our aim to introduce into the general theory of measure a concept of restricted measurability ${ }^{1}$ and to establish certain conditions under which it is equivalent to measurability in the familiar Carathéodory sense.

We follow the theory with two applications.

2. Notations and conventions. If $C$ is a sequence of sets and $n$ is an integer, we agree that

$$
\bigcup_{j=0}^{-1} C_{j}, \quad \bigcup_{j=0}^{n} C_{j}, \quad \text { and } \quad \bigcup_{j=0}^{\infty} C_{j}
$$

are respectively 0 , the union of terms of $C$ numbered from 0 to $n$, and the union of all terms of $C$.

We agree that a family $H$ of sets is hereditary if each subset of each member of the family is a member of the family and is countably additive if the union of members of each countable sub-family of $H$ is a member of $H$. By $\sigma H$ we mean the union of all members of $H$.

If $A$ and $B$ are sets, we agree that $A \sim B$ is the set of points in $A$ and not in $B$.

Throughout this paper we consider $S$ to be a fixed set with respect to which we make definitions.

3. Restricted measurability.

3.1. Definition. $\phi$ is a measure if and only if $\phi$ is such a function on

$$
\underset{\beta}{E}(\beta \subset S) \text { to } \underset{t}{E}(0 \leqq t \leqq \infty)
$$

that

$$
\phi(A) \leqq \sum_{\beta \in H} \phi(\beta)
$$

whenever $H$ is such a countable family that

$$
A \subset \sigma H \subset \text { S. }
$$

Since empty sums are zero, we see that, if $\phi$ is a measure, then:

I. $\phi(0)=0$;

II. $\phi(A) \leqq \phi(B)$ whenever $A \subset B C S$;

Received by the editors November 6, 1947.

1 A. P. Morse has contributed much to the development of this theory. 
III. $\phi(\sigma H) \leqq \sum_{\beta \in H} \phi(\beta)$ whenever $H$ is such a countable family that $\sigma H \subset S$.

3.2. Definition. $A$ is $\phi$ measurable $F$ if and only if $\phi$ is a measure and, for each member $B$ of $F, \phi(T B)=\phi(T B A)+\phi(T B \sim A)$ whenever $T \subset S$.

It is rather evident that a set, $\phi$ measurable $F$, is $\phi$ measurable $F^{\prime}$ where $F^{\prime}$ is the family of subsets of members of $F$.

In accordance with Carathéodory, we agree that $A$ is $\phi$ measurable if and only if $A$ is $\phi$ measurable

$$
\underset{\beta}{E}(\beta \subset S) .
$$

Following the next definition is the principal theorem of this paper.

3.3 Definition. $F$ is $\phi$ convenient if and only if $\phi$ is a measure, $F$ is hereditary, and, corresponding to each $T$ of finite $\phi$ measure, there exists such a sequence $C$ that

$$
\phi\left(T \sim \bigcup_{j=0}^{\infty} C_{j}\right)=0,
$$

and, for each integer $n$,

$$
C_{n} \subset C_{n+1} \in F, C_{n} \text { is } \phi \text { measurable } F .
$$

3.4. TheOREM. If $F$ is $\phi$ convenient, then $A$ is $\phi$ measurable whenever $A$ is $\phi$ measurable $F$.

Proof. If $\phi(T)=\infty$, then, clearly, $\phi(T)=\phi(T A)+\phi(T \sim A)$.

Suppose $\phi(T)<\infty$. We secure such a sequence $C$ that, for each integer $n$,

$$
\begin{aligned}
& C_{-1}=0, \text { and } \phi\left(T \sim \bigcup_{j=0}^{\infty} C_{j}\right)=0 . \\
& C_{n} \subset C_{n+1} \in F, C_{n} \text { is } \phi \text { measurable } F .
\end{aligned}
$$

We note that, for each integer $j, C_{j} \sim C_{j-1} \in F$.

Now, for each integer $n$ and $T \subset \mathcal{C S}$, we have $\phi\left(T C_{n}\right)=\phi\left(T C_{n} C_{n-1}\right)+\phi\left(T C_{n} \sim C_{n-1}\right)=\phi\left(T C_{n-1}\right)+\phi\left(T C_{n} \sim C_{n-1}\right)$.

By induction we obtain, for each integer $n$,

$$
\begin{gathered}
\phi(T) \geqq \phi\left(T C_{n}\right)=\sum_{j=0}^{n} \phi\left(T C_{j} \sim C_{j-1}\right)=\sum_{j=0}^{n} \phi\left\{T\left(C_{j} \sim C_{j-1}\right) A\right\} \\
+\sum_{j=0}^{n} \phi\left\{T\left(C_{j} \sim C_{j-1}\right) \sim A\right\} .
\end{gathered}
$$


Hence,

$$
\begin{aligned}
\phi(T) \geqq & \sum_{j=0}^{\infty} \phi\left\{T\left(C_{j} \sim C_{j-1}\right) A\right\}+\sum_{j=0}^{\infty} \phi\left\{T\left(C_{j} \sim C_{j-1}\right) \sim A\right\} \\
\geqq & \phi\left\{\bigcup_{j=0}^{\infty} T\left(C_{j} \sim C_{j-1}\right) A\right\}+\phi\left\{\bigcup_{j=0}^{\infty} T\left(C_{j} \sim C_{j-1}\right) \sim A\right\} \\
= & \phi\left\{T A \bigcup_{j=0}^{\infty} C_{j}\right\}+\phi\left\{(T \sim A) \bigcup_{j=0}^{\infty} C_{j}\right\} \\
& +\phi\left\{T A \sim \bigcup_{j=0}^{\infty} C_{j}\right\}+\phi\left\{(T \sim A) \sim \bigcup_{j=0}^{\infty} C_{j}\right\} \\
\geqq & \phi(T A)+\phi(T \sim A) \geqq \phi(T),
\end{aligned}
$$

where the third step follows from (1).

The proof is complete.

3.5. REMARK. The next definition and two obvious theorems following it enable us by using extant measure theory ${ }^{2}$ to avoid imitative direct methods for proofs that the complement of a set $\phi$ measurable $F$ and the union and intersection of a countable family of sets $\phi$ measurable $F$ are $\phi$ measurable $F$ and consequently to establish 3.9.

The notion of intersection measure is also useful in proving 4.2.

3.6. Definition. sect $(\phi, B)$ is the function $f$ on

$$
\underset{\beta}{E}(\beta \subset \mathcal{S})
$$

such that $f(\alpha)=\phi(\alpha B)$ for $\alpha \subset \mathcal{S}$.

3.7. TheOREM. If $\phi$ is a measure, then, for each $B$, sect $(\phi, B)$ is a measure.

3.8. Theorem. $A$ is $\phi$ measurable $F$ if and only if $\phi$ is a measure and, for each member $B$ of $F, A$ is sect $(\phi, B)$ measurable.

3.9 TheOREM. If $H$ is a family of sets $\phi$ measurable $F$ and $B$ is the intersection of all countably additive, $\mathcal{S}$ complemental families which contain $H$, then each member of $B$ is $\phi$ measurable $F$.

4. Applications. For Theorems 4.1 through 4.6 we assume that $\rho$ metrizes $S$ and agree that

$$
\operatorname{dist}(A, B)=\inf _{x \in A} \inf _{y \in B} \rho(x, y) \text {. }
$$

2 Measure theoretic results we use can be found in $\mathrm{H}$. Hahn, Theorie der reellen Funktionen, vol. 1, Berlin, 1921, pp. 424-432. 
Due to Caratheodory is the following well known theorem.

4.1. THEOREM. If $\phi$ is a measure and $\phi(A \cup B)=\phi(A)+\phi(B)$ whenever dist $(A, B)>0$, then each open set is $\phi$ measurable.

Stronger than 4.1 is 4.3 below.

4.2. Theorem. If $F$ is hereditary and $\phi(A \cup B)=\phi(A)+\phi(B)$ whenever $A$ and $B$ are such members of $F$ that dist $(A, B)>0$, then each open set is $\phi$ measurable $F$.

Proof. Suppose dist $\left(A^{\prime}, B^{\prime}\right)>0$. From the hereditariness of $F$ we see that

$$
\operatorname{sect}(\phi, T)\left(A^{\prime} \cup B^{\prime}\right)=\operatorname{sect}(\phi, T)\left(A^{\prime}\right)+\operatorname{sect}(\phi, T)\left(B^{\prime}\right)
$$

whenever $T \in F$. By 4.1 each open set is $\phi$ measurable $F$.

4.3. THEOREM. If $F$ is $\phi$ convenient and $\phi(A \cup B)=\phi(A)+\phi(B)$ whenever $A$ and $B$ are such members of $F$ that dist $(A, B)>0$, then each open set is $\phi$ measurable.

Proof. Use 3.4 and 4.2 .

An application of the foregoing is the following theorem.

4.4. ThEOREM. If $\phi$ is a measure and $\phi(A \cup B)=\phi(A)+\phi(B)$ whenever $A$ and $B$ are such bounded sets that dist $(A, B)>0$, then each open set is $\phi$ measurable.

Proof. Let $F$ be the family of bounded subsets of $S$. We establish the $\phi$ convenience of $F$ by taking a sequence of open spheres of integer radii the union of whose terms is $S$ and inferring from 4.2 the $\phi$ measurability $F$ of these spheres. Reference to 4.3 completes the proof.

4.5. Remark. The above theorem, which was communicated to me by A. P. Morse, instigated the present investigation. Morse proved it directly using a method of alternate annular shells.

I know of no correspondingly direct proof of the following application of 4.3.

4.6. THEOREM. If $\phi$ is a measure, $R$ is a sequence of non-dense sets,

$$
F=\underset{\beta}{E}\left(\beta \subset \mathcal{S} \text { and } \beta \bigcup_{j=0}^{\infty} R_{i} \text { is non-dense }\right),
$$

$\phi(A \cup B)=\phi(A)+\phi(B)$ whenever $A$ and $B$ are such members of $F$ that dist $(A, B)>0$, then each open set is $\phi$ measurable.

Proof. Let $C$ be such a sequence that, for each integer $n$, 


$$
C_{n}=\bigcup_{j=0}^{n}\left(\text { closure } R_{j}\right) \cup\left\{S \sim \bigcup_{j=0}^{\infty}\left(\text { closure } R_{j}\right)\right\} .
$$

Clearly, for each integer $n$,

$$
\bigcup_{j=0}^{\infty} C_{j}=S . \quad C_{n} \subset C_{n+1} \in F,
$$

After checking the hereditariness of $F$, we infer from 4.2 that each open set is $\phi$ measurable $F$. Hence, if we recall $3.5, C_{n}$ is $\phi$ measurable $F$ for each integer $n$. Thus $F$ is $\phi$ convenient. Reference to 4.3 completes the proof.

University of CaLIFornia

\section{ON THE DISTRIBUTION OF THE VALUES OF $|f(z)|$ IN THE UNIT CIRCLE}

\section{ROBERT BREUSCH}

1. Summary. Let $f(z)=1+a_{1} z+\cdots$ be analytic for $|z| \leqq 1$, $f(z) \not \equiv 1$. Then $|f(z)|$ will be greater than 1 at some points of the unit circle, and less than 1 at others. Calling $A(f)$ the area of the set of points within the unit circle, for which $|f(z)| \geqq 1$, let $\alpha$ and $\beta$ be the two largest non-negative constants such that $\alpha \leqq A(f) \leqq \pi-\beta$, for every $f(z)$. It is shown that $\alpha=\beta=0$; in other words, if $\epsilon$ is arbitrarily small positive, there are functions $f(z)$ such that $A(f)<\epsilon$, and others such that $A(f)>\pi-\epsilon$. The same is true, if $f(z)$ is restricted to polynomials $\prod_{\nu=1}^{n}\left(z-z_{\nu}\right)$ with $\coprod_{\nu=1}^{n}\left|z_{\nu}\right|=1$. These statements will be proved in $\$ 2$. $\$ 3$ contains a few additional results, given without proofs.

2. Proofs. The statements made in the summary are contained in the following theorem.

THEOREM. Let $P$ stand for the set of polynomials over the complex field of the form $f(z)=\prod_{\nu=1}^{n}\left(z-z_{\nu}\right)$, with $\prod_{\nu=1}^{n}\left|z_{\nu}\right|=1$; let $A(f)$ denote the area of the set of points in the unit circle, for which $|f(z)| \geqq 1$; let $\epsilon$ be an arbitrarily small positive number. Then $P$ contains polynomials $f_{1}(z)$ and $f_{2}(z)$ such that $A\left(f_{1}\right)>\pi-\epsilon$, and $A\left(f_{2}\right)<\epsilon$. 1948.

Presented to the Society, December 31, 1947; received by the editors January 7, 